\title{
A network analysis of post-traumatic stress and psychosis symptoms
}

Article

Accepted Version

Hardy, A., O'Driscoll, C., Steel, C., van der Gaag, M. and van den Berg, D. (2021) A network analysis of post-traumatic stress and psychosis symptoms. Psychological Medicine, 51 (14). pp. 2485-2492. ISSN 1469-8978 doi:

https://doi.org/10.1017/S0033291720001300 Available at https://centaur.reading.ac.uk/91411/

It is advisable to refer to the publisher's version if you intend to cite from the work. See Guidance on citing.

To link to this article DOI: http://dx.doi.org/10.1017/S0033291720001300

Publisher: Cambridge University Press

All outputs in CentAUR are protected by Intellectual Property Rights law, including copyright law. Copyright and IPR is retained by the creators or other copyright holders. Terms and conditions for use of this material are defined in the End User Agreement.

\section{www.reading.ac.uk/centaur}

\section{CentAUR}

Central Archive at the University of Reading 
Reading's research outputs online 
Running title: Post-traumatic stress in psychosis

\section{A Network Analysis of Post-traumatic Stress and Psychosis Symptoms}

Amy Hardy ${ }^{* 1,2}$, Ciaran O'Driscoll $^{3}$, Craig Steel ${ }^{4}$, Mark van der Gaag, ${ }^{5,6}$, David van den Berg ${ }^{5,6}$

${ }^{1}$ Department of Psychology, Institute of Psychiatry, Psychology \& Neuroscience, King's College London, De Crespigny Park, SE5 8AF, U.K.

2 South London \& Maudsley NHS Foundation Trust, Bethlem Royal Hospital, Monks Orchard Road, Beckenham, Kent, BR3 3BX, U.K.

${ }^{3}$ Research Department of Clinical, Educational and Health Psychology, University College London, Gower Street, London, WC1E 6BT, U.K.

${ }^{4}$ The Oxford Institute of Clinical Psychology Training, Oxford, U.K.

${ }^{5}$ VU University, Department of Clinical Psychology and Amsterdam Public Health Research, van der Boehorsttraat 7, 1081 BT Amsterdam, The Netherlands.

${ }^{6}$ Parnassia Psychiatric Institute, Zoutkeetsingel 40, 2512 HN Den Haag, Netherlands.

Abstract word count: 250

Total word count: 3974

\section{Funding}

This work was supported by the National Institute for Health Research (NIHR) for the C-PAS Trial, under its Research for Patient Benefit (RfPB) Programme (Grant Reference Number PBPG-1207-15077). The views expressed are those of the authors and not necessarily those of the NHS, the NIHR or the Department of Health. The T.TIP trial was supported by the Dutch support foundation Stichting tot Steun Vereniging voor Christelijke Verzorging van Geestes en Zenuwzieken (Dr van der Gaag). CO'D is supported by the UCLH NIHR BRC.

*Corresponding Author:

Dr Amy Hardy, Department of Psychology, Institute of Psychiatry, Psychology \& Neuroscience, De Crespigny Park, SE5 8AF.

Tel: $+44(0) 2078485178$

Fax: +44 (0)207848 5006

Email: amy.hardy@kcl.ac.uk 
Running title: Post-traumatic stress in psychosis

\section{Abstract}

Background: Understanding the interplay between trauma-related psychological mechanisms and psychotic symptoms may improve the effectiveness of interventions for post-traumatic stress reactions in psychosis. Network theory assumes that mental health problems persist not because of a common latent variable, but from dynamic feedback loops between symptoms, thereby addressing the heterogeneous and overlapping nature of traumagenic and psychotic diagnoses. This is a proof-of-concept study examining interactions between post-traumatic stress symptoms, which were hypothesized to reflect trauma-related psychological mechanisms, and auditory hallucinations and delusions.

Method: Baseline data from two randomised controlled trials $(\mathrm{N}=216)$ of trauma-focused therapy in people with post-traumatic stress symptoms (87.5\% met PTSD diagnostic criteria for PTSD) and psychotic disorder were analysed. Reexperiencing, hyperarousal, avoidance, trauma-related beliefs, auditory hallucinations and delusional beliefs were used to estimate a Gaussian graphical model along with expected node influence and predictability (proportion of explained variance).

Results: Trauma-related beliefs had the largest direct influence on the network and together with hypervigilance, were implicated in the shortest paths from flashbacks to delusions and auditory hallucinations.

Conclusions: These findings are in contrast to previous research suggesting a central role for re-experiencing, emotional numbing and interpersonal avoidance in psychosis. Traumarelated beliefs were the psychological mechanism most associated with psychotic symptoms, although not all relevant mechanisms were measured. This work demonstrates that investigating multiple putative mediators may clarify which processes are most relevant to trauma-related psychosis. Further research should use network modelling to investigate how the spectrum of traumatic stress reactions play a role in psychotic symptoms. 
Running title: Post-traumatic stress in psychosis

\section{Introduction}

It is now accepted that psychosis is influenced by social factors. Trauma is 3ypothesized to play a causal role in the occurrence and phenomenology of psychosis for a subgroup of people (Bebbington, 2015; Okkels et al., 2017; Varese et al., 2012). This opens up new possibilities for preventing and managing the devastating impact that psychosis can have on people's lives (Schizophrenia Commission, 2012). Given that resilience is the most common trajectory following trauma, we need to develop our understanding of the role of trauma-related mechanisms in distressing psychosis (Galazer-Levy et al., 2018; Hardy, 2017; Holmes et al., 2018). These mechanisms can then be targeted in interventions, in line with a causal interventionist approach (Goekoop \& Goekoop, 2014).

Progress will be hampered if research is constrained by diagnostic constructs (Brewin \& Patel, 2010). This is because there is marked phenomenological, symptomatic and diagnostic overlap in psychosis and traumagenic mental health problems, such as depression and Post-Traumatic Stress Disorder (PTSD) (Geddes et al., 2016; McCarthy-Jones \& Longden, 2015; Okkels et al., 2017). Further, PTSD and Schizophrenia-spectrum diagnoses have been criticised as they are highly heterogenous categories. This heterogeneity suggests that different causal mechanisms may be involved for any given individual and that subtypes of trauma-related psychosis may exist (Bentall et al., 2014; Galatzer-Levy \& Bryant, 2018).

Network theory can address this complexity, as it assumes that mental health difficulties arise not from a common latent variable, but from dynamic feedback loops between components ('nodes') which are investigated using network analysis (Borsboom, 2017; Fried et al., 2016) The feedback loops are the result of causal interactions between nodes ('edges') that are maintained in a network. The effect of any given node within a network varies by strength, with certain nodes playing a relatively more central role. As network nodes often cut across diagnostic boundaries, network analysis provides a means of developing a transdiagnostic understanding of how post-traumatic stress and psychosis symptoms interact. Further, certain interactions may cluster, potentially allowing for the identification of hypothesized subtypes of trauma-related psychosis. To date, no studies have investigated post-traumatic stress and psychosis symptoms using network analysis. We therefore describe a proof-of-concept exploratory study examining the interactions between these symptoms, and any and any clusters within them, to inform future research.

Network analyses relevant to this study have tended to focus on either trauma and 
Running title: Post-traumatic stress in psychosis

psychosis, or PTSD. For example, Fried et al. (2018) compared four moderate to large samples with PTSD (with civilian, refugee, combat and professional-related traumas) and found that intrusive memories, hyperarousal and emotional detachment were among the most central symptoms. Similarly, intrusive memories, together with negative emotions and beliefs, were most central at baseline and 3-year follow-up in 311 military veterans with PTSD (Von Stockert et al., 2018). It is of note that these central symptoms include both the hallmark symptoms of PTSD (e.g. intrusive memories and hyperarousal) and depression (e.g. negative emotions and beliefs, and emotional and behavioural detachment), in line with a transdiagnostic approach. Isvoranu et al. (2016) investigated the network associations between childhood trauma types and psychotic dimensions (i.e. positive, negative and general psychopathology) in 552 people with psychosis. In support of an affective pathway to psychosis, they identified that all relationships were connected through emotional and behavioural difficulties, with anxiety having a dominant role in the network. These findings indicate the potential utility of examining the interactions between psychosis and post-traumatic stress symptoms.

A drawback of network theory is that it has tended to focus on symptoms as network components (Borsboom, 2017). Building on recent proposals, we argue that to enhance the usefulness of network methods to psychological theory and therapy, empirically-based mechanisms (which may be reflected by symptoms or other psychosocial phenomenon) should be included as nodes (Fried \& Cramer, 2017; Payton et al., 2017). Hardy (2017) highlights current evidence suggesting the key trauma-related psychological mechanisms involved in psychosis are intrusive trauma memories, beliefs, and cognitive, behavioural and interpersonal emotion regulation. Whilst these putative trauma-related processes have been investigated in the literature, multiple mechanisms are rarely examined and as a result little is known about their relative associations with psychotic symptoms. Network analysis may help to elucidate which trauma-related variables are most related to psychotic symptoms. In this study, we focus on the associations between intrusive trauma memories (i.e. reexperiencing), emotional regulation (i.e. hyperarousal, avoidance and emotional numbing), beliefs (i.e. post-traumatic cognitions) and positive psychotic symptoms (i.e. auditory hallucinations and delusional beliefs). Our first two research questions aimed to highlight the most central symptoms in a sample of people with psychosis diagnoses, with the final question exploring whether symptom associations appeared to reflect distinct subtypes of trauma-related psychosis. The following questions were examined: 
Running title: Post-traumatic stress in psychosis

1. Which symptoms are most influential in a network of post-traumatic stress and psychosis symptoms?

2. Which symptoms account for identified associations in a network of post-traumatic stress and psychosis symptoms?

3. Are there identifiable sub-clusters of associations in post-traumatic stress and psychotic symptoms?

\section{Method}

Baseline data from two randomized controlled trials of trauma-focused therapy for people with post-traumatic stress symptoms and psychosis diagnoses were combined for the retrospective analysis (Cognitive Behaviour Therapy for PTSD in Schizophrenia (C-PAS) trial, Steel et al., 2017a, and Treating Trauma in Psychosis (T.TIP) trial, van den Berg et al., 2015). The C-PAS trial was given ethical approval by Berkshire Research Ethics Committee (SC/09/ H0505/85) and was registered as ISTCRN 67096137 and the T.TIP trial was approved by the medical ethics committee of the VU University Medical Center and was registered as ISRCTN 79584912.

\section{Participants}

The combined sample $(\mathrm{N}=216)$ represents a broad spectrum of individuals with psychosis diagnoses and post-traumatic stress symptoms. Both trials recruited adult participants (age $18-65)$, with a current or lifetime diagnosis of psychotic disorder from community psychosis teams, with sufficient language to participate and with no diagnosed organic impairment. Symptom variability is a requisite of network analysis which was supported by differences in the PTSD and psychosis diagnostic criteria for the two trials. For PTSD symptoms, the C-PAS trial participants reflected a broader range of PTSD symptom severity because $45 \%(n=27)$ did not meet diagnostic criteria for PTSD. Psychotic symptom variability was ensured as the C-PAS trial criterion was current schizophrenia-spectrum disorder whereas the T.TIP trial criterion was lifetime diagnosis of a psychotic disorder. Sample characteristics are displayed in Table 1, further information on the sample and recruitment methods is published in the original papers. 
Running title: Post-traumatic stress in psychosis

\section{Measures and variables}

Measures were selected based on which assessments were included in both trials. Variables were then extracted from three measures. Data was taken from baseline assessments.

The Clinician-Administered PTSD Scale (CAPS) (Blake et al., 1995)

The CAPS is a semi-structured interview based on the DSM-IV (APA, 1994) criteria for Posttraumatic Stress Disorder. There are 17 symptoms items including five for re-experiencing, five for hyperarousal and seven for avoidance and emotional numbing. Each item is scored on two 5-point scales (0 to 4) for frequency and intensity that are then summed to produce a total item score ranging from 0 to 8 , or a total severity score ranging from 0 to 136 . The 17 individual item scores were used in this study. As described above, these symptoms were conceptualised as reflecting trauma-related psychological mechanisms (i.e. re-experiencing was a proxy for episodic trauma memory intrusions, and hyperarousal and avoidance and numbing symptoms as types of emotional regulation). The CAPS is a valid and reliable measure with high specificity and sensitivity, and is a widely used interview for diagnosing PTSD in psychosis (Blake et al., 1995; Mueser et al., 2001).

Post Traumatic Cognitions Inventory (PTCI) (Foa et al., 1999)

The PTCl is a 36-item self-report questionnaire that assesses post-traumatic cognitions. These were viewed as reflecting the psychological mechanism of trauma-related beliefs. Scores range from 1 to 7 and are summed into three factors, which were used in the analysis: negative cognitions about self (21 items), negative cognitions about the world (seven items), and self-blame (five items). The PTCI has been found to have strong psychometric properties (Foa et al., 1999).

The Psychotic Symptom Rating Scales (PSYRATS) (Haddock et al., 1999)

The PSYRATS is a semi-structured interview that assesses the phenomenology of auditory hallucinations and delusional beliefs. For the purposes of this study, the frequency/intensity factor items were used as they best captured the psychological constructs of interest (Woodward et al., 2014). For auditory hallucinations, these were the summed scores of frequency, duration, and disruption items and for delusional beliefs they included the sum of the preoccupation, conviction, and disruption items. The PSYRATS scales have excellent 
Running title: Post-traumatic stress in psychosis

inter-rater reliability and have been found to have strong validity (Haddock et al., 1999).

\section{Network Analysis}

The statistical software R (version 3.5) was used to conduct statistical analyses. Packages used included qgraph (Epskamp et al., 2012), bootnet (Epskamp et al., 2018), mgm (Haslbeck \& Waldorp, 2018) and networktools (Jones, 2017). The network modelling approach taken followed an Extended Bayesian Information Criterion (EBIC) least absolute shrinkage and selection operator (LASSO) procedure, implemented within a single Gaussian random fields network. This method controls model complexity through the model selection process and subjecting estimated regression parameters to a penalty (small associations are reduced to zero). Thus producing a model with good prediction accuracy and sparse representation of the predictors in the model (Zou et al., 2017). Missing cases were handled with pairwise deletion. The variables were ordinal but were not normally distributed as such a nonparanormal transformation was applied to network items to relax assumptions of normality in our dataset. Within the graphical representation, 'edges' are the lines between nodes representing regularized partial correlations; the estimated relationship between two variables while controlling for all other variables. These associations represent predictive associations (similar to multiple regression). The presence of an edge indicates a dependent relationship between variables, the absence indicates that they are conditionally independent (given all other nodes in the network). Blue edges illustrate positive associations and red edges negative associations. The wider and more saturated the edge, the stronger the association. The accuracy of edges and stability estimates for the network were calculated using a bootstrapping procedure (1000 iterations) (Epskamp \& Fried, 2018).

Our first research question was examined through calculation of centrality metrices of expected influence (Haslbeck \& Waldorp, 2018) and predictability (Jones, 2017) for each item. Expected influence reflects the total level of involvement of a node in the network. One-step expected influence is defined as the sum of all edge strengths extending from a given node (local centrality), whereas two-step expected influences assesses centrality up to two edges away from the node (global centrality). A node could be highly central but share nearly no variance with other nodes. As such we have also calculated predictability which quantifies how much influence we can have on a node by intervening on all its neighbours: item variance 
Running title: Post-traumatic stress in psychosis

predicted by connected items $\left(R^{2}\right)$. If a node displays high centrality and predictability, this supports the interpretation of the importance of this node within the network.

To address our second research question, we computed the shortest path (Dijkstra, 1959) from flashbacks (the hallmark symptom of PTSD, Brewin et al., 2010; Brewin, 2015) to positive psychotic symptoms (auditory hallucinations and delusions) within the network. This analysis identified the nodes (symptoms) which mediate the predictive quality between them. We also included a bridge expected influence strength metric (Jones, 2017) to quantify symptoms which connect post-traumatic stress symptoms to positive psychotic symptoms. Identifying the bridging items between post-traumatic stress and positive psychotic symptoms used the $80^{\text {th }}$ percentile cut off on the scores of bridge expected influence metric.

For the third research question, a model-based, dimension reducing clustering approach (Scrucca, 2010) was taken to reveal subpopulations, given the expected heterogeneity in psychosis samples (e.g. Millan et al., 2014). R package Mclust version 5 (Scrucca et al., 2016) was used for analysis. A description of the modelling procedure is included in supplementary materials.

\section{Results}

Descriptive statistics

The descriptive statistics for the sample are shown in Table 1. The majority of participants had a schizophrenia diagnosis and scored above the threshold for severe Post-traumatic Stress Disorder (CAPS >65, Mueser et al., 2001) (further information on the T.TIP and C-PAS samples is provided in Table 1 in supplementary materials and Steel et al, 2017a, 2017b; van den Berg et al, 2015).

Table 1: Sample demographics and symptom descriptives $(N=216)$

\begin{tabular}{lll}
\hline & & $M(S D) / n(\%)$ \\
\hline Age & & $41.46(10.36)$ \\
Gender & Male & $109(50.5)$ \\
& Female & $107(49.5)$ \\
& &
\end{tabular}


Running title: Post-traumatic stress in psychosis

\begin{tabular}{lll}
\hline Diagnoses & Schizophrenia & $140(64.8)$ \\
& Schizoaffective disorder & $61(28.2)$ \\
& Other psychosis & $5(2.3)$ \\
Symptoms & Mood disorder with psychotic features & $10(4.6)$ \\
& Auditory hallucinations (PSYRATS) & $12.62(14.47)$ \\
& Delusional beliefs (PSYRATS) & $10.23(7.99)$ \\
& PTSD (CAPS) & $68.18(16.96)$ \\
& PTCI & $151.7(33.98)$
\end{tabular}

Research question one: Which post-traumatic stress and psychosis symptoms have the strongest associations with each other?

The network (see Figure 1) yielded 82 positive (and 1 negative) relationships out of 231 possible edges. Edge and strength centrality stability were adequate $(0.44$ and 0.36 respectively) (see Figures $1-3$ in supplementary materials for stability analysis). Assessing expected influence revealed that negative cognitions about the self and negative cognitions about the world had the largest direct influence on the network (locally and globally) (see Figure 4 in supplementary materials). Predictability estimates (the proportion of item variance explained by pairwise interactions within the network) are displayed in Figure 1 (see Table 2 in supplementary materials). Predictability of nodes was low overall (mean of 22\%) Negative cognitions about self (52\%) and the world (43\%) could be best explained by associated nodes (including each other). Auditory hallucinations (18\%), trauma memory intrusions (21\%) and flashbacks (19\%) displayed relatively moderate predictability, and may be independent of other factors or determined by factors not included in the network.

Research question 2: Which symptoms account for associations between post-traumatic stress and psychosis?

Negative cognitions about the self, negative cognitions about the world, self-blame and hypervigilance bridged post-traumatic stress and psychosis symptoms in this sample (see Figure 5 in supplementary materials). The shortest path analysis (see Figure 2) displays the route from flashbacks to delusions and auditory hallucinations. Flashbacks and delusions are conditionally independent and indirectly connected through sense of a foreshortened future and negative cognitions about the world. The shortest pathway between flashbacks and 
Running title: Post-traumatic stress in psychosis

auditory hallucinations was indirectly connected by the same nodes, although also passed through delusions. Thus, the estimated models and metrics suggest that auditory hallucinations, delusions and flashbacks are conditionally independent and mediated by these associated variables.

Research question 3: Are there subgroups of associations between post-traumatic stress and psychosis symptoms?

To address the question of whether the data indicated subtypes of trauma-related psychosis, we examined whether there were sub-clusters of associations between post-traumatic stress and psychotic symptoms. We performed a dimensional reduction in order to explore possible clusters. A one-dimensional equal variance model supported a 2-class solution, which yielded a best fitting model with a BIC value of - 823.75 (see Figure 6 in supplementary materials). This model explained a large proportion of the variation across variables (eigenvalue $=0.757$ ) . This suggests the observed scores in the entire sample are not from a distinct severity population relate to two distributions, reflecting two subpopulations ( $n=70, n=146$ ). One group $(n=70)$ was characterised by significantly more severe PTSD symptoms, auditory hallucinations and trauma-related beliefs, although there were no differences between the groups for self-blame, amnesia and delusional beliefs. The sample size of each group precluded a reliable interpretation of networks, however the severe population form a distinct separate class in which the correlational structure is fundamentally different from the less severe population.

\section{Discussion}

This study is the first to use network analysis, which derives partial correlations, to examine the interplay between post-traumatic stress and psychotic symptoms. Trauma-related beliefs were the most influential symptoms, and together with hypervigilance, accounted for the shortest paths between flashbacks, delusions and hallucinations (with the latter only connected to re-experiencing through delusional beliefs). These results are in contrast to findings which suggest a central role for re-experiencing and dissociative detachment in hallucinations to explain the association between trauma and psychosis (Berry et al., 2017; Luhrmann et al., 2019; McCarthy-Jones \& Longden, 2016). Instead, this study suggests beliefs reflecting a lack of safety, control and self-esteem account for the interactions between PTSD 
Running title: Post-traumatic stress in psychosis

and psychosis. This is consistent with the emphasis on inter- and intrapersonal threat in theoretical models and research evidence (Freeman \& Garety, 2014)'. Threat beliefs are likely to interact with emotions, sensory-perceptual experiences, cognitive biases, appraisals, behaviour and interpersonal relating, thereby contributing to psychosis and PTSD (Brewin et al., 2010; Hardy, 2017).

Symptoms that can be conceptualised as linked to emotional regulation (e.g. loss of interest, interpersonal distance and hypervigilance) were the next most influential nodes in the main network. Further, hypervigilance was a bridge symptom between post-traumatic stress and psychotic symptoms. These findings are in line with previous network analyses of PTSD and suggest that understandable attempts to regulate threat may paradoxically perpetuate symptoms of post-traumatic stress and psychosis (Fried et al., 2018; Von Stockert et al., 2018). They are also consistent with the proposed role of selective attention for threat in paranoia, which is often not distinguishable from trauma-related hypervigilance (Freeman et al., 2013). Interestingly, trauma-related dissociative detachment as measured by emotional numbing (which can be conceptualised as an emotion regulation strategy, Brown, 2006;) did not appear to have a strong impact on psychosis, despite proposals implicating its role in voice hearing (Berry et al., 2017; Dalenberg et al., 2012; Luhrmann et al., 2019; McCarthy-Jones \& Longden, 2016; Pearce et al., 2017; Varese et al., 2012). This suggests that activation of the sympathetic nervous system (associated with flight and fight responses) may be more potent in maintaining psychotic experiences than parasympathetic activation (related to flag and freeze responses).

We found that intrusive trauma memories also had a less central relationship than has been previously reported in studies of PTSD and voice hearing (Fried et al., 2018; Alsawy et al., 2015). This finding is also consistent with the T.TIP trial which reported that effectively reducing re-experiencing did not significantly affect hallucination severity, although it did have a significant impact on paranoia and other symptoms of PTSD. The re-experiencing symptoms of emotional distress and physiological reactivity did have a relatively more central role in the network. However, this may be attributable to the activation of beliefs in autobiographical memory leading to trauma-congruent emotions and bodily responses, not to involuntary recollections of specific episodic memories. It may also be that the intrusion of event memories in psychosis occurs differently to re-experiencing in PTSD. Severely decontextualised trauma memories (i.e. whereby arousal has inhibited the encoding of the 
Running title: Post-traumatic stress in psychosis

spatial and temporal context of events) could manifest as intrusions which are experienced as occurring in the 'here and now' without any sense of a past experience being recollected, and so would not be experienced as memories (Hardy, 2017; Steel et al., 2005).

Two networks of interactions distinct from those in the main network were also identified, suggesting our sample may reflect two distinct subtypes of trauma-related psychosis. One was more severe and characterised by beliefs, re-experiencing, voices and delusions, whereas beliefs were most influential in the other, and occurred with delusions, interpersonal detachment, emotional reactivity and physiological reactivity. The possibility of a subtype of post-traumatic stress in psychosis typified by entrenched trauma-beliefs, that generalise to delusions and detachment, with another subtype also being maintained by intrusive memories and hallucinations, requires further investigation. It may be that the latter subgroup reflects the occurrence of complex PTSD with psychosis symptoms (Frost et al., 2019). (p.X)

Whilst sample size limits the reliability of this analysis and it requires replication, it provide a preliminary indication that distinct subgroups of symptom interactions may exist, which may plausibly require distinct therapeutic approaches (van den Berg \& Hardy, 2020). In relation to therapeutic targets, overall our analysis suggests modifying trauma-related beliefs may have a beneficial impact on both PTSD and psychosis, in line with evidence that they are key mechanism of change in trauma-focused therapy (Cooper et al., 2017). However, the C-PAS trial targeted trauma-related beliefs using cognitive restructuring to reduce PTSD in psychosis and obtained a null result on all outcomes (Steel et al., 2017). This illustrates that directly targeting the mechanism with the strongest impact on the network may not be an effective way to destabilise it (Bringmann et al., 2020). Given the high predictability of traumarelated beliefs, it could be more effective to intervene on the mechanisms which interact with them. In support of this, the T.TIP trial focused on modifying re-experiencing and avoidance using Prolonged Exposure and EMDR, and found these interventions were effective in reducing trauma-related beliefs, paranoia and other PTSD symptoms (van den Berg et al., 2015). As re-experiencing and avoidance are mechanistically linked to trauma-related beliefs and psychosis, it makes sense that targeting the former has a beneficial effect on the latter (Hardy, 2017). For example, flashbacks of a sexual assault and avoidance of memories may be maintained by beliefs that 'others will harm me' and 'I cannot cope', which also contribute to paranoia. By reducing avoidance and reprocessing the memories of sexual assault through 
Running title: Post-traumatic stress in psychosis

exposure techniques, the person may learn that 'not everyone will harm me' and 'I can manage'. These alternative trauma-related beliefs may then have a negative interaction with paranoia and reduce psychosis severity (van den Berg \& Hardy, 2020).

Whilst the study shows that the network approach holds promise for developing our understanding of post-traumatic stress and psychosis, there are important limitations. First, the sample was biased as it only included people with significant PTSD symptoms which may have limited its variance and meant it did not reflect the range of trauma-related presentations in psychosis (e.g. people with a solely negative symptom profile would not have been included). The base rate of symptoms may therefore account for the findings, and replication is needed in a more representative sample reflecting the full spectrum of posttraumatic stress reactions. The limited strength of associations between some nodes (including auditory hallucinations and re-experiencing) possibly indicates that not all the relevant mechanisms were included. Other potential psychological mechanisms include emotions, metacognitive beliefs and a broader range of emotional regulation strategies, which could be investigated alongside micro (e.g. neurochemical) and macro (e.g. social) processes (Isvoranu et al., in press). This study did not include some commonly used measures of trauma-related processes, and the assessment of dissociative detachment being restricted to emotional numbing and not including depersonalisation and derealisation is a significant limitation. The low predictability may also be the result of the heterogeneous nature of symptoms. If distinct subtypes of trauma-related psychosis exist, they are likely to be associated with different causal processes and associations with post-traumatic stress symptoms (McCarthy-Jones et al., 2016). We emphasise the hypotheses we have outlined from the findings require further investigation, as causal inferences are not possible given the study's cross-sectional design, the modest network stability, and the undetermined flow of mechanisms in the network (Fried et al., 2017; Bringmann et al., 2018). Time series investigation of the role of trauma-related mechanisms in psychosis is required to improve our understanding of the dynamic interactions between post-traumatic and psychotic symptoms. Indeed, it has been argued that focusing on the overall dynamics of a network may be more useful in identifying intervention targets than trying to analyse the most central network nodes, given that modifying these may not be effective in destabilising the network Bringmann et al., 2018). A limitation of the analysis is that EBIC tuning parameter (set to zero), can lead to the estimation of false positive associations emphasising sensitivity (at the 
Running title: Post-traumatic stress in psychosis

cost of specificity). While the absence of an edge may reflect the absence of a true association it may also reflect insufficient power to identify a small association. Confirmation of the results in a large sample using a more conservative LASSO tuning parameter is warranted.

In summary, this is the first study to examine the interplay of post-traumatic stress and psychotic symptoms using network analysis. To investigate the potential role of traumarelated psychological processes in psychosis, re-experiencing symptoms were conceptualised as reflecting the involuntary retrieval of episodic trauma memories, hyperaroused, avoidant and numbing symptoms as types of emotional regulation, and post-traumatic cognitions as schematic beliefs. In line with trauma-focused theories of psychosis and evidence from therapy trials, the findings support the role of cognitive, emotional, behavioural and interpersonal processes in psychosis (Hardy, 2017; van den Berg et al., 2015). Whilst the approach is in its infancy, network models may provide a compelling opportunity to tailor micro, meso and macro level interventions to target the specific processes underlying the development and maintenance of problems (Borsboom, 2017, Fried \& Cramer, 2017, Looijestijn et al., 2015). The network identified in this study suggests that building a sense of safety, control and self-worth could reduce the widespread influence of trauma-related beliefs on symptoms. Longitudinal investigations incorporating comprehensive assessment of putative mechanisms are now needed to advance our understanding of post-traumatic stress in psychosis, and support the development of effective interventions.

\section{Acknowledgements}

We would like to thank all the participants, clinicians and researchers involved in the T.TIP and C-PAS trials for supporting this work. AH, CS and DvB receive payment for training and supervision on post-traumatic stress in psychosis. MvG and CO'D declare no conflicts of interest in relation to this work.

\section{References}

Alsawy S, Wood L, Taylor PJ and Morrison AP. (2015) Psychotic experiences and PTSD: associations in a population survey. Psychological Medicine 20, 1-11.

Berry K, Varese F and Bucci S. (2017) Cognitive attachment model of voices: Evidence base and future implications. Frontiers in Psychiatry 8, 111.

Brewin CR, Gregory JD, Lipton M and Burgess N. (2010) Intrusive images in psychological 
Running title: Post-traumatic stress in psychosis

disorders: characteristics, neural mechanism, and treatment implications. Psychological Review 117(1), 210- 232.

Bebbington PE. (2015) Unravelling psychosis: Psychosocial epidemiology, mechanism and meaning. Shanghai Archives of Psychiatry 27, 70-81.

Bentall R, de Sousa P, Varese F et al. (2014) From adversity to psychosis: pathways and mechanisms from specific adversities to specific symptoms. Social Psychiatry and Psychiatric Epidemiolology 49, 1011-1022.

Blake DD, Weathers FW, Nagy LM, et al. (1995) The development of a Clinician-Administered PTSD Scale. Journal of Traumatic Stress 8, 75-90.

Borsboom D. (2017) A network theory of mental disorders. World Psychiatry 16, 5-13.

Brand RM, McEnery C, Rossell S, Bendall S and Thomas N. (2018) Do trauma-focussed psychological interventions have an effect on psychotic symptoms? A systematic review and meta-analysis. Schizophrenia Research 195, 13-22.

Brewin CR. (2015) Re-experiencing traumatic events in PTSD: new avenues in research on intrusive memories and flashbacks. Eur. J. Psychotraumatol 6, doi: 10.3402/ejpt.v6.27180.

Brewin CR, Gregory JD, Lipton M and Burgess N. (2010) Intrusive images in psychological disorders: characteristics, neural mechanism, and treatment implications. Psychol. Rev. 117(1), 210-232.

Brewin CR, Patel T. (2010) Auditory pseudohallucinations in United Kingdom war veterans and civilians with posttraumatic stress disorder. Journal of Clinical Psychiatry 71(4), 419-425. Bringmann L, Elmer T, Epskamp S et al. (2019) What do centrality measures measure in psychological networks? J Abnorm Psychol. 128(8), 892-903.

Brown RJ. (2006) Different types of "dissociation" have different psychological mechanisms. J. Traumatic Dissociation. 7(4), 7-28.

Cooper AA, Clifton EG and Feeny NC. (2017). An empirical review of potential mediators and mechanisms of prolonged exposure therapy. Clinical Psychology Review, 138(3), 106-121. Dalenberg CJ, Brand BL, Gleaves DH, Dorahy MJ, Loewenstein RJ, Cardena E, Frewen PA, Carlson EB and Spiegel D. (2012). Evaluation of the evidence for the trauma and fantasy models of dissociation. Psychological Bulletin, 138(3), 550-588.

Dijkstra EW. (1959) A note on two problems in connexion with graphs. Numerische Mathematic 1(1), 269-271. 
Running title: Post-traumatic stress in psychosis

Epskamp S, Borsboom D and Fried El. (2018) Estimating psychological networks and their accuracy: A tutorial paper. Behavioural Research Methods 50(1), 195-212.

Epskamp S, Cramer AOJ, Waldorp L, Schmittmann VD and Borsboom D. (2012) qgraph: Network Visualizations of Relationships in Psychometric Data. Journal of Statistical Software 48(4), 1-18.

Epskamp S, Fried E I. (2018) A tutorial on regularised partial correlation networks. Psychological Methods 23(4), 617-634.

Foa EB, Ehlers A, Clark DM, Tolin DF and Orsillo SM. (1999) The posttraumatic cognitions inventory (PTCI): Development and validation. Psychological Assessment 11(3), 303-314.

Freeman D and Garety P. (2014) Advances in understanding and treating persecutory delusions: a review. Social Psychiatry and Psychiatric Epidemiology 49(8), 1179-1189.

Freeman D, Thompson C, Vorontsova $\mathbf{N}$ et al. (2013) Paranoia and post-traumatic stress disorder in the months after a physical assault: A longitudinal study examining shared and differential predictors. Psychological Medicine 43(12), 2673-2684.

Luhrmann TM, Alderson-Day B, Bell V et al. (2019) Beyond trauma: A multiple pathways approach to auditory hallucinations in clinical and non-clinical populations. Schizophrenia Bulletin 45(Suppl 1), S35-S42.

Frost R, Louison Vang M, Karatzias T, Hyland P and Shevlin M. (2019) The distribution of psychosis, ICD-11 PTSD and complex PTSD symptoms among a trauma-exposed UK general population sample, Psychosis, 11:3, 187-198.

Fried EI, van Borkulo CD, Cramer AO, Boschloo L, Schoevers RA and Borsboom D. (2016) Mental disorders as networks of problems: a review of recent insights. Social Psychiatry and Psychiatric Epidemiology 52(1), 1-10.

Fried EI, Eidhof MB, Palic S et al. (2018) Replicability and generalizability of posttraumatic stress disorder (PTSD) networks: a cross-cultural multisite study of PTSD symptoms in four trauma patient samples. Clinical Psychological Science 6(3), 335-351.

Fried E and Cramer AOJ. (2017) Moving forward: Challenges and directions for psychopathological network theory and methodology. Perspectives in Psychological Science 12(6), 999-1020.

Galatzer-Levy IR and Bryant RA. (2018) 636,120 Ways to Have Posttraumatic Stress Disorder. Perspectives in Psychological Science 8(6), 651-662. 
Running title: Post-traumatic stress in psychosis

Galatzer-Levy IR, Huang SH and Bonanno GA. (2018) Trajectories of resilience and dysfunction following potential trauma: A review and statistical evaluation. Clinical Psychology Review 63, 41-55.

Geddes G, Ehlers A and Freeman D. (2016) Hallucinations in the months after a trauma: An investigation of the role of cognitive processing of a physical assault in the occurrence of hallucinatory experiences. Psychiatry Research 246, 601-605.

Goekoop R and Goekoop JG. (2014) A network view on psychiatric disorders: network clusters of symptoms as elementary syndromes of psychopathology. PLoS One 9, e112734.

Hardy A. (2017) Pathways from trauma to psychotic experiences: A theoretically informed model of posttraumatic stress in psychosis. Frontiers in Psychology 8, 697.

Haddock G, McCarron J, Tarrier N and Faragher EB. (1999) Scales to measure dimensions of hallucinations and delusions: the psychotic symptom rating scales (PSYRATS). Psychological Medicine 29(4), 879-889.

Haslbeck JMB and Waldorp L. (2018) mgm: Structure Estimation for Time-Varying Mixed Graphical Models in high-dimensional Data. arXiv preprint:1510.06871v2.

Holmes E, Ghaderi A, Harmer JC et al. (2018) The Lancet Psychiatry Commission on psychological treatmnents research in tomorrow's science. Lancet Psychiatry 5(3), 237-286.

Isvoranu AM, Borsboom D, van Os J and Guloksuz S. (2016) A network approach to environmental impact in psychotic disorders: Brief theoretical framework. Schizophrenia Bulletin 42(2), 870-873.

Isvoranu AM, Boyette LL, Guloksuz S and Borsboom D. (in press) Symptom Network Models of Psychosis. In CA Tammina, E Ivleva, U Reininghaus and J van Os. Dimensions of Psychosis. Oxford University Press: Oxford.

Kendler KS and Campbell J. (2009) Interventionist causal models in psychiatry: repositioning the mind-body problem. Psychological Medicine 39(6), 881-7.

Looijestijn J, Blom JD, Aleman A et al. (2015) An integrated network model of psychotic symptoms. Neuroscience and Biobehavioural Review 59, 238-250.

McCarthy-Jones S and Longden E. (2015) Auditory verbal hallucinations in schizophrenia and post-traumatic stress disorder: common phenomenology, common cause, common interventions? Frontiers in Psychology 6, 1071.

McCarthy-Jones S, Thomas N, Strauss C et al. (2014) Better than mermaids and stray dogs? Subtyping auditory verbal hallucinations and its implications for research and 
Running title: Post-traumatic stress in psychosis

practice. Schizophrenia Bulletin 40(Suppl_4), S275-S284.

Mueser KT, Salyers MP, Rosenberg SD, Ford JD, Fox L and Carty P. (2001) Psychometric evaluation of trauma and posttraumatic stress disorder assessments in persons with severe mental illness. Psychological Assessment 13, 110-117.

Okkels N, Trabjerg B, Arendt M and Pedersen CB. (2017) Traumatic stress disorders and risk of subsequent schizophrenia spectrum disorder or bipolar disorder: a nationwide cohort study. Schizophrenia Bulletin 43, 180-186.

Jones P. (2017) networktools: Tools for identifying important nodes in networks. R package. Payton J, Heeren A and McNally RJ. (2017) Commentary: A network theory of mental disorder. Frontiers in Psychology 8, 1305.

Pearce J, Simpson J, Berry K, Bucci S, Moskowitz A and Varese F. (2017) Attachment and dissociation as mediators of the link between childhood trauma and psychotic experiences. Clinical Psychology and Psychotherapy 24(6), 1304-1312.

Pilton M, Varese F, Berry K and Bucci S. (2015) The relationship between dissociation and voices: A systematic literature review and meta-analysis. Clinical Psychology Review 40, 138155.

Scrucca L. Dimension reduction for model-based clustering. Computational Statistics 20(4), 471-484.

Millan MJ, Fone K, Steckler T and Horan WP. (2014) Negative symptoms of schizophrenia: clinical characteristics, pathophysiological substrates, experimental models and prospects for improved treatment. European Neuropsychopharmacology 24(5), 645-692.

Scrucca L, Fop M, Murphy TB and Raftery AE. (2016) mclust 5: Clustering, classification and density estimation using gaussian finite mixture models. $R$ J 2016:8(1);289.

Steel C, Fowler D and Holmes EA. (2005) Traumatic intrusions in psychosis: an information processing account. Behavioural and Cognitive Psychotherapy 33, 139-152.

Steel C, Hardy A, Smith B et al. (2017) Cognitive-behaviour therapy for post-traumatic stress in schizophrenia: a randomized controlled trial. Psychological Medicine 47(1), 43-51.

Steel C, Doukani A, Hardy A. (2017b) The PCL as a brief screen for posttraumatic stress disorder within schizophrenia. International journal of psychiatry in clinical practice, 21(2), 148-150. 
Running title: Post-traumatic stress in psychosis

The Schizophrenia Commission. (2012) The abandoned illness: a report from the Schizophrenia Commission. London: Rethink Mental Illness.

van den Berg DPG, de Bont PA, van der Vleugel BM et al. (2015) Prolonged exposure vs eye movements desensitization and reprocessing vs waiting list for posttraumatic stress disorder in patients with a psychotic disorder: a randomised clinical trial. JAMA Psychiatry 72(3), 259267.

van den Berg DPG and Hardy A. (2020) Chapter 19: Trauma-focused therapies for psychosis. In J. Badcock \& G. Paulik, A Clinical Handbook of Psychosis. Elsevier.

Varese F, Barkus E and Bentall RP. (2012) Dissociation mediates the relationship between childhood trauma and hallucination proneness. Psychological Medicine 42, 1025-1036.

Varese F, Smeets F, Drukker M et al. (2012) Childhood adversities increase the risk of psychosis: a meta-analysis of patient-control, prospective- and cross-sectional cohort studies. Schizophrenia Bulletin 38, 661-671.

Von Stockert SHH, Fried El, Armour C and Pietrzak RH. (2018) Evaluating the stability of DSM5 PTSD symptom network structure in a national sample of U.S. military veterans. Journal of Affective Disorders 229, 63-68.

Woodward TS, Jung K, Hwang H et al. (2014) Symptom dimensions of the psychotic symptom rating scales in psychosis: a multisite study. Schizophrenia Bulletin 40(Suppl_4), S265-S274.

Zou H, Hastie T and Tibshirani R. (2017) On the "degrees of freedom" of the lasso. Annals of Statistics 35(5), 2173-2192. 
Figure 1. The network graph shows associations and predictability estimates between posttraumatic stress symptoms (i.e. re-experiencing, avoidance and numbing, hyperarousal), post-traumatic cognitions, and positive symptoms of psychosis (i.e. auditory hallucinations and delusional beliefs). The edges represent strength of association between nodes (used to calculate expected influence), with the shaded area of the pie chart around the nodes indicating the predictability measure (i.e. dark segment representing higher predictability).

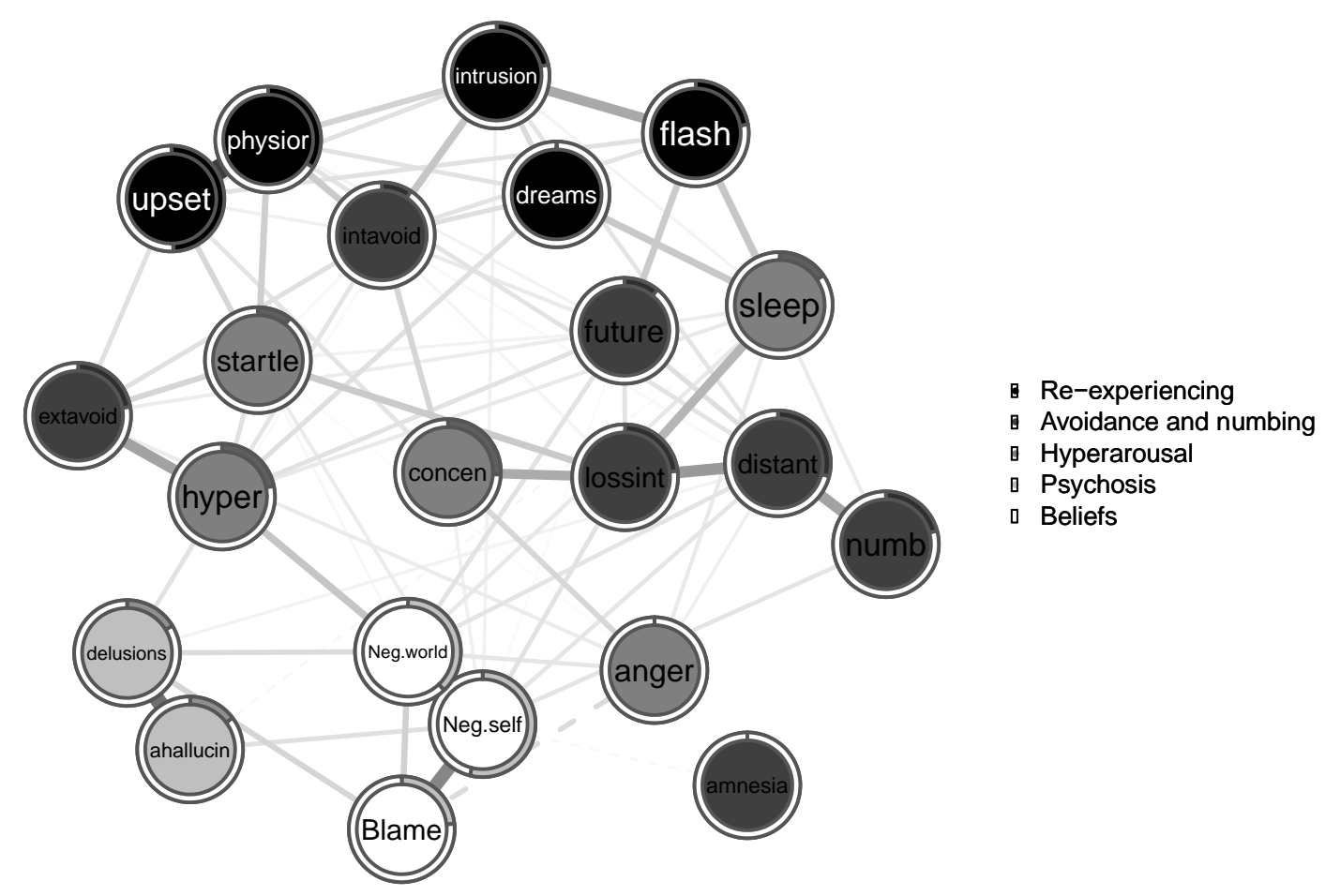

\footnotetext{
* Dreams $=$ recurrent distressing dreams, physior $=$ physiological reactivity to trauma cues, upset $=$ psychological distress to trauma cues, intrusions = intrusive distressing trauma recollections, flash = acting or feeling as if the traumatic event were recurring, extavoid $=$ avoiding trauma-related activities, places or people, intavoid $=$ avoiding trauma-related thoughts, feelings and conversations, lossint $=$ diminished interest or participation in activities, future $=$ sense of foreshortened future, distant $=$ feeling of detachment or estrangement from others, numb $=$ restricted range of affect, amnesia $=$ inability to recall important parts of trauma, hyper $=$ hypervigilance, startle $=$ exaggerated startle response, sleep $=$ difficulty falling or staying asleep, concen $=$ difficulty concentrating, anger = irritability or outbursts of anger, negworld = negative beliefs about world, negself $=$ negative beliefs about self, blame $=$ self-blame, ahallucin $=$ frequency/intensity of auditory hallucinations, delusions $=$ frequency/intensity of delusions.
} 
Running title: Post-traumatic stress in psychosis

Figure 2: Bridging symptoms between post-traumatic stress and and positive psychotic symptoms are highlighted in green, and shortest paths between flashbacks, auditory hallucinations and delusions are shown.

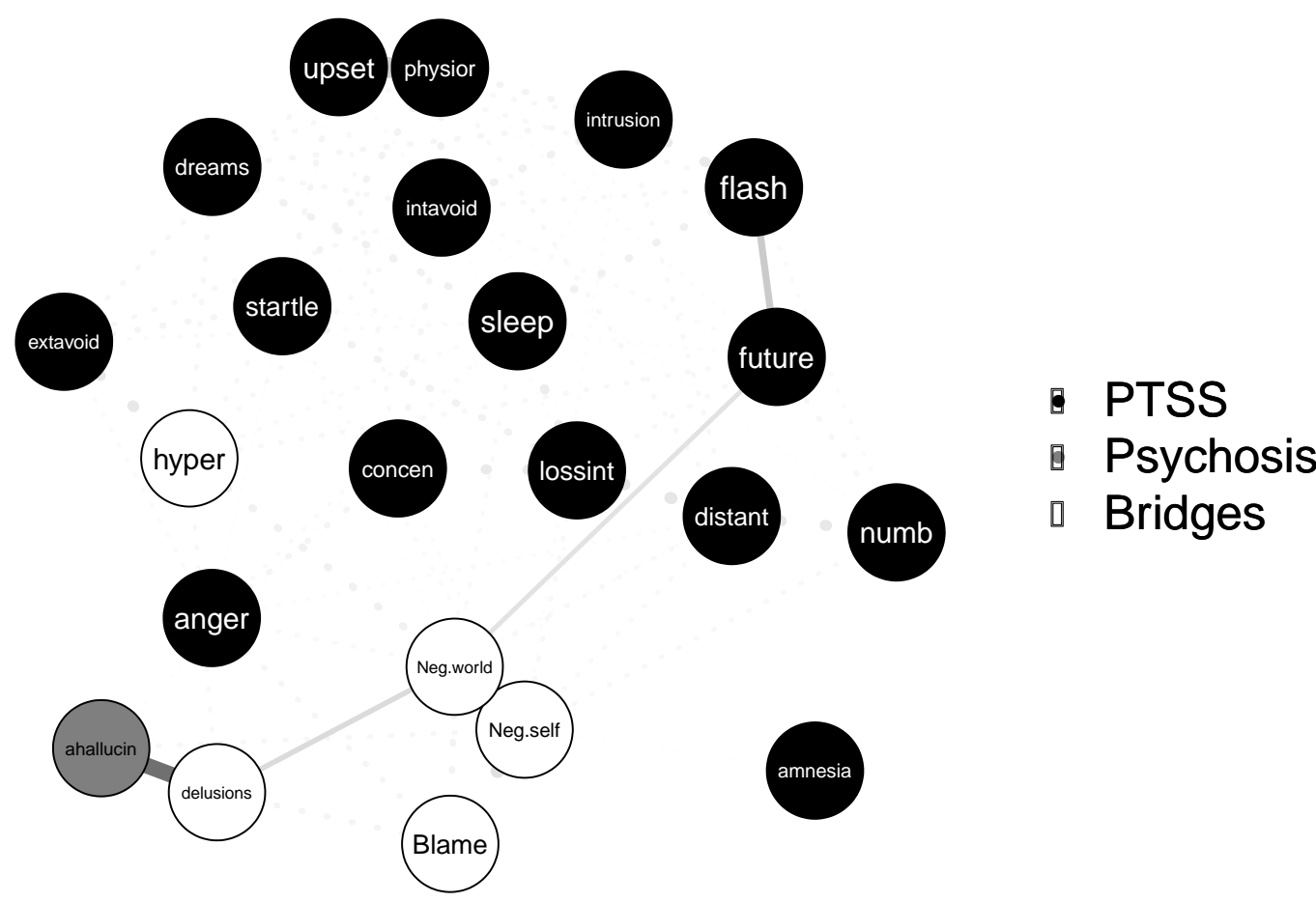

* Dreams $=$ recurrent distressing dreams, physior $=$ physiological reactivity to trauma cues, upset $=$ psychological distress to trauma cues, intrusions = intrusive distressing trauma recollections, flash $=$ acting or feeling as if the traumatic event were recurring, extavoid $=$ avoiding trauma-related activities, places or people, intavoid = avoiding trauma-related thoughts, feelings and conversations, lossint $=$ diminished interest or participation in activities, future $=$ sense of foreshortened future, distant $=$ feeling of detachment or estrangement from others, numb = restricted range of affect, amnesia = inability to recall important parts of trauma, hyper $=$ hypervigilance, startle $=$ exaggerated startle response, sleep $=$ difficulty falling or staying asleep, concen = difficulty concentrating, anger $=$ irritability or outbursts of anger, negworld $=$ negative beliefs about world, negself $=$ negative beliefs about self , blame $=$ self-blame, ahallucin $=$ frequency/intensity of auditory hallucinations, delusions $=$ frequency/intensity of delusions. 